\title{
INTEGRATED HETEROGENEOUS SOCIAL NETWORKING SITES
}

\author{
Kirti Jadhav $^{1}$, Kiran Esave ${ }^{2}$, Bhakti Gholap ${ }^{3}$, M. G. Devikar ${ }^{4}$ \\ ${ }^{1}$ Student, MESCOE - Pune, State-Maharashtra, Country-India \\ ${ }^{2}$ Student, MESCOE - Pune, State-Maharashtra, Country-India \\ ${ }^{3}$ Student, MESCOE - Pune, State-Maharashtra, Country-India \\ ${ }^{4}$ Professor, MESCOE - Pune, State-Maharashtra, Country-India
}

\begin{abstract}
The growth of Social Network Sites (SNSs) have opened the opportunity for researchers to explore a large amount of social and behavioral data. The heterogeneity of SNSs offer more seamless services across SNSs and also offers integrated SNSs. The aim of the system is to integrate the heterogeneous SNSs. The P2P-iSN allows users from heterogeneous SNSs to communicate without involving the SNS they have registered with. In proposed system architecture, Global Relationship Model (GRM) is used to capture the relationship strength, and $i$-Search, to find the optimal social path with less time between any two users who are meaningfully connected in heterogeneous SNSs. With the P2P-iSN, SNS developers can design more effective user-centric SNS application. All SNS user uses different services of different SNS on a single platform, and maintain relationship globally.
\end{abstract}

Keywords-SNS , GRM, OpenID, GlobalID, i-search

\section{INTRODUCTION}

Today Social Networking Sites (SNS) have become a part of our day to day life. They helped us to make the world smaller and integrated with each other. There are many SNS available today and many more are piling each day [2]. Thus users use many SNSs to share data with friends and family.

As a result, a user may register with multiple SNSs for different social network applications, carry multiple SNS accounts, interact with contacts from different SNSs, publish and access different web contents, and share contents within each SNS community. While SNSs offer different services, one key feature shared among SNSs is how they are built around users and users' existing social networks [3], [4]. Yet each SNS is isolated, so users manage their profiles and build relationship separately on different SNSs. The content for the same user in different SNSs may overlap, so it becomes a burden for users to manage contents across different SNSs.

With growing influence and complexity of SNSs, researchers have been developing methods to connect users and aggregating data across SNSs so that each SNS no longer stands alone. Here, we propose system architecture to integrate heterogeneous SNSs and maintain the social relationships among a large number of users across heterogeneous SNSs. For example, imagine that within an SNS user $b$ is in user $a$ 's friend list. There is a directional social link denoted by " $a \rightarrow b$ " from user $a$ to user $b$. When there exists a social path between two users in an SNS, then there is a "relationship" between these two users. We say that there is a "global relationship" from user $a$ and user $b$ if there is a social path from $a$ to $b$ over heterogeneous SNSs. By identifying "global relationship" among users over heterogeneous SNSs, the system allows different SNSs to interconnect with various networks. Integrated heterogeneous SNSs provide different services of different SNSs over a single platform and establishes path between two users with less time.

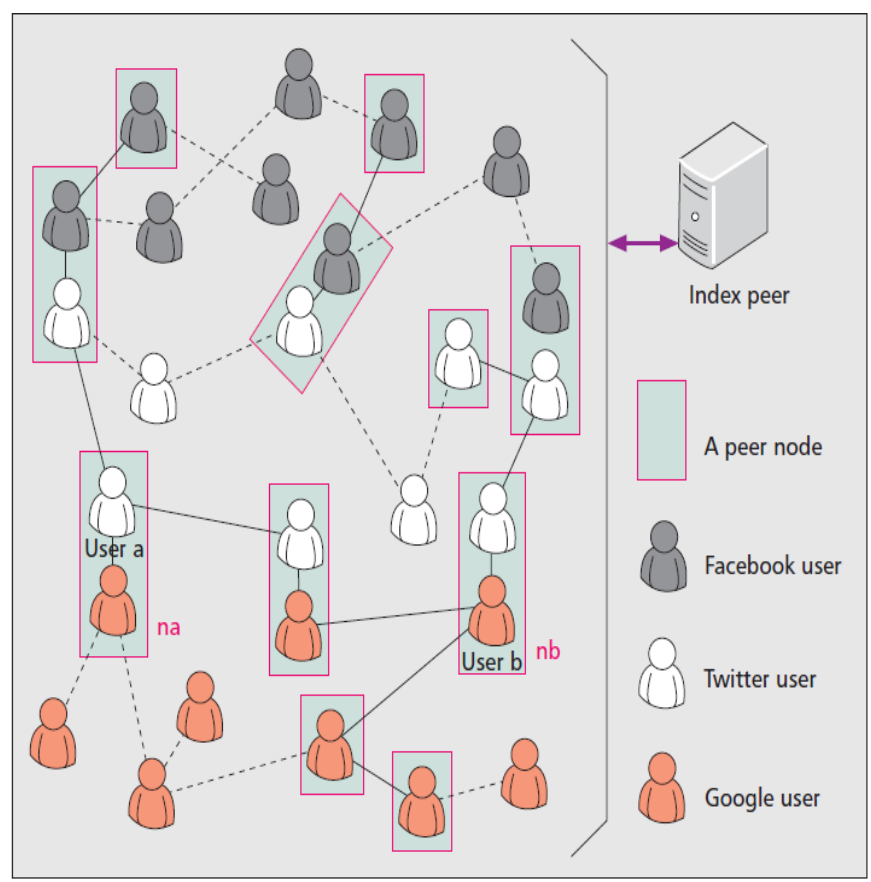

Fig .1 System architecture of the proposed P2P-iSN.

In above figure the operations over P2P-iSN. When a peer node is turned on, it reports to the index peer node the online status, which consists of its ID and IP address of the peer node. Upon receiving the online status, the index peer node updates the online status for the peer node.

If a user $a$ of the peer node na and a user $b$ of the peer node $\mathbf{n b}$ are on each other's friend list in a SNS, and na and $\mathbf{n b}$ are turned on, these two online peer nodes can communicate 
with each other by using the corresponding IP addresses queried from the index peer node. The peer nodes can establish social paths among users from different SNSs and build the so-defined "global relationship."

With the peer-to-peer network architecture, P2P-iSN allows users from heterogeneous SNSs to communicate without involving any specific SNS, and the integration is independent of any specific SNS. By applying P2P-iSN, we develop a Global Relationship Model to assess the strength of the global relationship between two users from heterogeneous SNSs. Based on the global relationship model, we propose a searching mechanism, namely $i$ Search, to find the social path between two users from heterogeneous SNSs.

\section{P2P-iSN}

In this section, we propose a peer-to-peer architecture, namely $P 2 P-i S N$, to integrate heterogeneous SNSs. P2P-iSN consists of two kinds of nodes: peer nodes and index peer nodes.

\subsection{Peer Node}

A peer node is installed on an end device for a user to access SNSs, and its main functionality is to integrate heterogeneous SNSs. The user of a peer node may register to one or more SNSs on his end-device, and possibly login to one or more SNSs at the same time. To associate these different accounts of the same user from heterogeneous SNSs, a unique user ID may be required. The concept known as OpenID [6] can serve this purpose although any other uniquely identifiable ID can be used. A unique user ID can be some kind of authenticated information like user's cell phone number or verifiable email address. The main functionality of a peer node is to integrate the heterogeneous SNSs through the Friend List maintenance. Peer nodes communicate with each other directly and form a peer-topeer network. The phone book in a peer node (e.g., Jenny's end device) is used as the base to integrate heterogeneous SNSs. Take Fig. 2a as an example. Jenny has a friend John with phone number "0910456."

A database, friend list, is maintained to store the information about a user's friends. Figure $2 \mathrm{~b}$ shows the format of a friend list. The friend list consists of three kinds of information: personal information, social network information, and address information.

Personal information field stores the IDs of the user's friends, including the ID in SNS, phone number, and email address. In different SNSs, users may use different IDs. As shown in Fig. 2b, for example, Jenny's friend, John, uses the ID, "John_f", on Facebook TM and use the ID, "John_t", on Twitter $\left.{ }^{\mathrm{TM}}\right)^{-}$. The phone number associates the entry in the phone book with the entry in the friend list. An entry in the phone book may be mapped to multiple entries in the friend list.
Social Network Information field consists of four subfields, including SN Type, T Value, Timestamp, and Online. The SNS Type indicates which SNS the friend has registered. For example, in Figure 2b, Jenny's friend, John, registered to Facebook ${ }^{\mathrm{TM}}$ using ID "John_f." The T Value stores the result calculated by using Eq. 1 in the global relationship model that will be elaborated later, indicating the frequency that a user performs some kind of social activities on his friend (e.g., Jenny posts a comment, click a "Like", or send a message on John's wall in Facebook ${ }^{\mathrm{TM}}$ ).

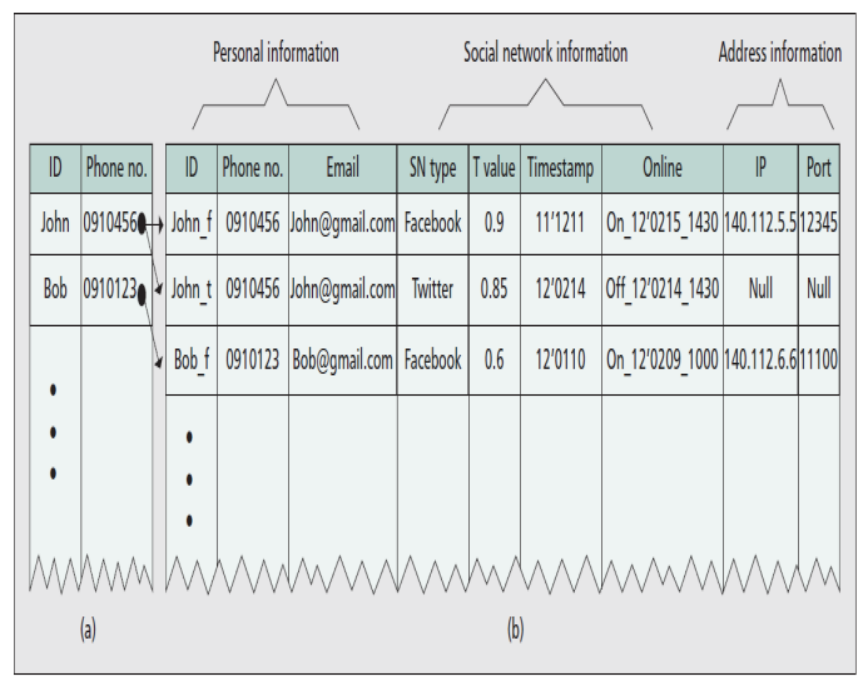

Fig.2 An example of friend list; a)Jenny's phone book; b) Jenny's friend list

For example, in (1) in Figure 2b, the $\mathrm{T}$ Value for Jenny $\leftarrow$ John on Facebook ${ }^{\mathrm{TM}}$ is 0.9 . The Timestamp field stores the time when the $\mathrm{T}$ Value was calculated. The Online indicates that whether the friend is on the SNS now or not and when John logins to the Facebook ${ }^{\mathrm{TM}}$ last time. If the value of Online is "On" ("Off"), the time is when John logins (logouts) Facebook ${ }^{\mathrm{TM}}$. For example, in Figure 2b, "On 12'0215 1430" implies that John f logins Facebook ${ }^{\mathrm{TM}}$ at 14:00 on Feb. 15, 2012, and is now on Facebook ${ }^{\mathrm{TM}}$.

Address Information field stores the IP address and the port number of the friend's end-device. This information is valid when the peer node of the friend is turned on.

\subsection{Index Peer Node}

The index peer node is responsible for maintaining the status (i.e., online or off-line) and the routing information (i.e., IP address) of each peer node. When a peer node is turned on, it reports to the index peer node the online status, which consists of its ID and IP address of the peer node. Upon receiving the online status, the index peer node updates the online status for the peer node. 


\begin{tabular}{|c|c|c|c|c|c|}
\hline ID & Phone no. & Email & $\mathbb{I P}$ & Port & SN type \\
\hline John__ & 0910123 & John@gmail.com & 140.112 .5 .5 & 12345 & Facebook \\
\hline Bob_f & 0910456 & Bob@gmail.com & 140.112 .6 .6 & 11100 & Facebook \\
\hline Bob_t & 0910456 & Bob@gmail.com & 140.112 .6 .6 & 11100 & Twitter \\
\hline Jenny_f & 0910789 & Jenny@gmail.com & 140.112 .7 .7 & 16161 & Facebook \\
\hline Jenny_t & 0910789 & Jenny@gmail.com & 140.112 .7 .7 & 16161 & Twitter \\
\hline - & & & & & \\
\hline - & & & & & \\
\hline
\end{tabular}

Fig.3 An example of Global ID list

An index peer node is a database that maintains the GlobalID list as shown in figure .For each online peer node, an entry is created in the GlobalID List for the peer node. Similar to the Friend List, the GlobalID List consists of three kinds of information: Personal Information, Social Network Information, and Address Information for an online user.

Turning on Peer Node. This section describes the execution of a peer node. When a user turns on the peer node on his end device, the Login procedure is executed. Figure shown below illustrates the message flow for the Login procedure with the following steps:

Step 1. When a user turns on the peer node, then function is executed to authenticate the user in an SNS.

Step 2. If the authentication is successful, the SNS responds with the user SNS ID.

Step 3. The peer node creates send a message (i.e., the User_Online_Message message) carrying the user's ID, Phone No., Email, IP address, port number, and SN Type, to the index peer node. The index peer node creates an entry for the user in the global ID list.

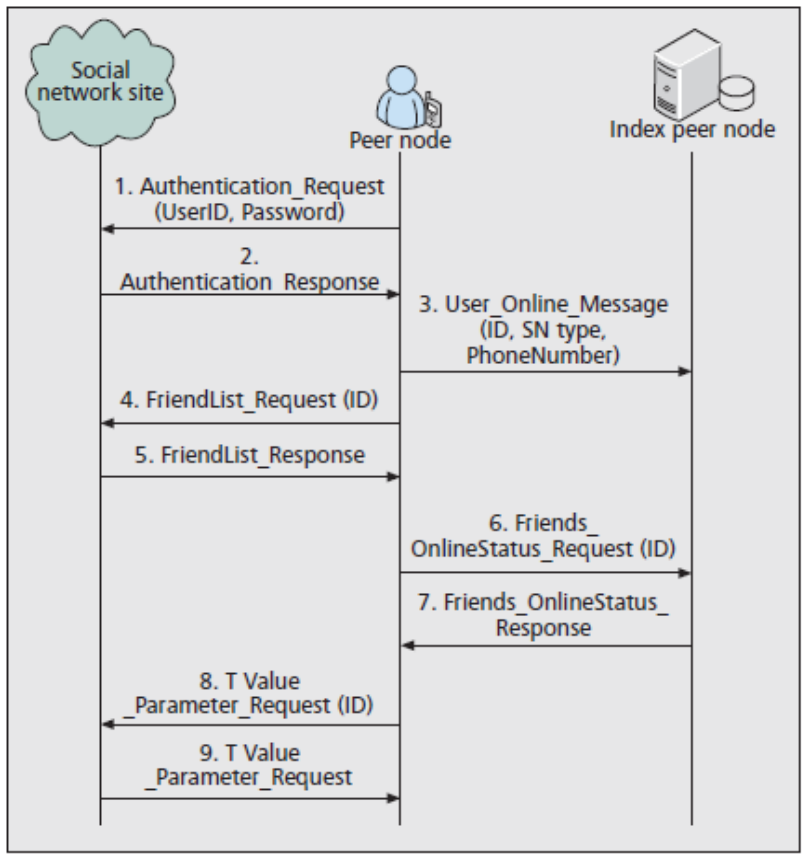

Fig. 4 The message flow for login procedure

Steps 4 and 5. The peer node sends a request or response (i.e., the FriendList_Request and FriendList_Response message pair) to get the IDs of the user's friends from the SNSs, and creates an entry for each friend in the Friend List. Steps 6 and 7. The peer node send a message (i.e., the Friends_OnlineStatus_Request and Friends_OnlineStatus_ Response message pair) to the index peer node to query the online friends of the user.

Steps 8 and 9. The peer node collect the social activity information to calculate the $\mathrm{T}$ value from the SNS by exchanging the T Value_Parameter_Request and the $\mathrm{T}$ Value_Parameter_Response message pair.

\section{GRM (GLOBAL RELATIONSHIP MODEL)}

This model is used to identify global relationship between two users across heterogeneous SNSs. It is tool to measure the Global relationship strength between two users. A directional social link $a \rightarrow b$ is associated with frequency, which is denoted by $f(a, b)$, to capture how often a user $a$ performs some kind of social activities with user $b$ (e.g., $a$ posts a comment on $b$ 's wall, click a "Like", send a message, or makes a call to $b$ ). Consider there are $C$ kinds of social activities. For $1 \leq i \leq C$, let $\lambda_{\mathrm{i}}$ denote the frequency that a user $a$ performs the ith kind of activity with user $b$. We define $f(a, b)$ by

$$
f(a, b)=\sum_{i=1}^{c} w_{i} \lambda_{i}
$$

where $w_{i}$ is the weight for the ith kind of activity, $0 \leq w_{i} \leq 1$ for $1 \leq i \leq C$, and $\sum_{\mathrm{i}=1}^{\mathbf{c}} w_{i}=1$. Note that in eq (1) the weight $w_{i}$ is a fine-tuning tool to reflect different degrees of interactions in relationship. 
An interaction factor exists between user $a$ and user $b$ if $a \rightarrow$ $b$ and $b \rightarrow a$ exist, and the value $F(a, b)$ for an interaction factor is defined by

$$
F(a, b)=\left(\frac{\min \{f(a, b\}), \theta}{\theta}\right)\left(\frac{\min \{f(b, a)\}, \theta}{\theta}\right)
$$

A larger $F(a, b)$ means more interactions between user $a$ and user $b$, and refer to "(2)", we have $0 \geq F(a, b) \leq 1$ and $F(a, b)=F(b, a)$.

In propose system a function $Z(\mathbf{P})$ to measure the strength of the global relationship between $u 1$ and $u L+1$, which is defined by

$$
Z(P)=\left\{\begin{array}{lr}
1 & \text { if } L=0 \\
\prod_{i=1}^{L} F\left(u_{i} u_{i+1}\right) & \text { atherwise }(i, e \mathbb{L} \geq 1)
\end{array}\right.
$$

A larger $Z(\mathbf{P})$ implies stronger global relationship. The strength $Z(\mathbf{P})$ is a powerful tool that can provide more precise friend recommendation and trust/reputation metrics, and also serve as a basis for content sharing across SNSs.

\section{4. i-Search ALGORITHM}

In this section, we propose i-Search mechanism to find a directional social path between two peer nodes in P2P-iSN. The i-Search mechanism establishes social paths link by link. When a link is added into a path, global relationship strength is calculated for the new path using the $Z$ (.) function in Eq. 3. If the global relationship strength for the new path is below a threshold $\Delta$, the social path search stops. Note that $\Delta$ is used to guarantee that the global relationship strength for the constructed path is strong enough so that users are motivated to use the global social relationship for further SNS applications.

The past social network research findings in the sociology indicated that [11] $F(a, b)=0.5$. However this may not be true anymore for online social networks. In online social networks, $F(a, b)$ would be much high than 0.5 . In this study, for demonstration purpose, we set up $\Delta$ based on the research findings in the sociology (i.e., the interaction factor for link

$a \rightarrow b$ is $F(a, b)=0.5)$. If we consider a path $\mathbf{P}$ with length $|\mathbf{P}|=4$, then using the $Z$ (.) function using (3), the global relationship strength for the path is $Z(\mathbf{P})=0.5^{4}=0.0625$, which is considered very weak relationship. Therefore we set $\mathrm{D}=0.5^{3}=0.125$.

Details of the i-Search mechanism are given below: The index peer node maintains the online status (including the ID and IP address of the peer node) for the online peer nodes. A friend list is maintained in the peer node, which stores the online information for all friends of the peer node. To simplify our description, we use "the friend $b$ of a peer node $a$ " to imply that the social link $a \rightarrow b$ exists. When a peer node is turned on, it reports its online status to the index peer node, and receives the latest online status for his friends from the index peer node. With the latest online information, the peer node can determine whether his friend is online (i.e., a peer node is turned on). A online peer node can communicate with his online friends directly. We run a recursive algorithm, iSearch, in the peer node (Algorithm 1). In this algorithm, the set $\mathbf{G}$ is the friend list of a peer node. The input parameter s stores the ID of the peer node who calls Algorithm 1, and $r$ is the ID of the peer node to be searched. Initially, we set $\mathbf{P} \leftarrow \boldsymbol{\emptyset}$.

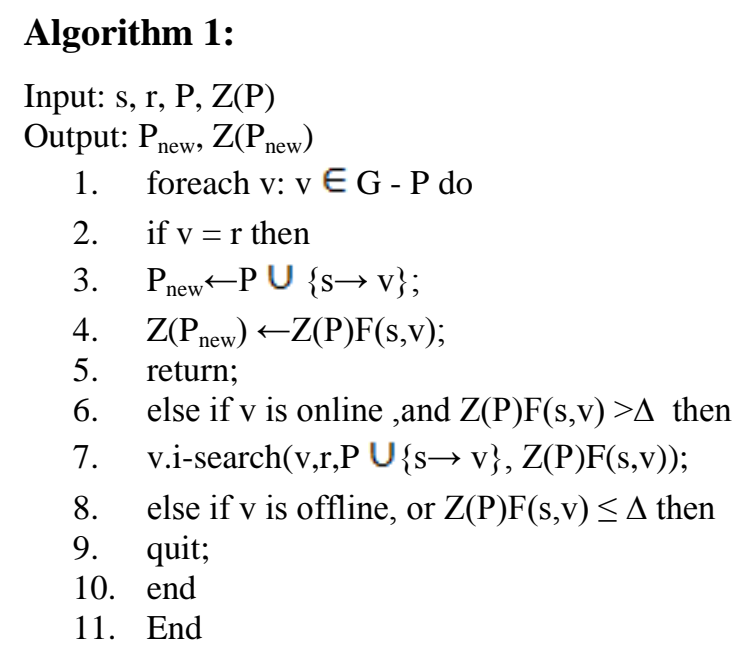

Consider the scenario where user a register on index peer node after registration peer node shows his friend's status (Online/Offline) then user can directly access his own SNS without login overhead. For direct communication between peer node $\mathrm{a}$ and peer node $\mathrm{b}$ both user $\mathrm{a}$ and $\mathrm{b}$ should registered on index peer node. A user $a$ can "request" his friend $b$ to execute the iSearch algorithm (i.e.,iSearch() in Algorithm 1) through the direct communication if $b$ is online. That is, the directional social path $\mathbf{P}$ is established among the peer nodes. Note that the i-Search mechanism may find multiple global social relationships between two peer nodes. For the peer node who triggers the i-Search mechanism, he can use the one with the largest global social relationship strength. Furthermore, we can speed up the execution of the i-Search mechanism by caching the searching results on the peer nodes.

\section{CONCLUSION}

In this paper, we propose $\mathrm{P} 2 \mathrm{P}-\mathrm{iSN}$, a peer-to-peer network architecture to integrate multiple SNSs without incurring excessive overhead to the SNSs. With integrated model, we could develop an effective approach, a Global Relationship Model, to evaluate the global relationship strength between two users with more precision. With $\mathrm{P} 2 \mathrm{P}-\mathrm{iSN}$ and the Global Relationship Model as the foundation, we propose the i-Search mechanism to find the social path with certain level of social relationship strength in a P2P social network. Our proposed approach can find the desired social path with high probability comparing to traditional approach and can effectively establish global social relationship for users from heterogeneous SNSs. 


\section{REFERENCES}

[1] P2P-iSN: A Peer-to-Peer Architecture for Heterogeneous Social Networks Phone Lin and PaiChun Chung, National Taiwan University Yuguang Fang, University of Florida.

[2] C. Zhang, "Privacy and Security for Online Social Networks: Challenges and Opportunities," IEEE Network, vol. 24, no. 4, July/Aug. 2010, pp. 13-18.

[3] A. Mislove, "Measurement and Analysis of Online Social Networks," Proc. 7th ACM SIGCOMM Conf. Internet Measurement, 2007,pp.29-42.

[4] N. Ellison and D. Boyd, "Social Network Sites: Definition, History, and Scholarship," J. ComputerMediated Communication, vol. 13, no. 1, Oct.

[5] M. N. Ko , "Social-Networks Connect Services," Computer, vol. 43, no. 8, Aug. 2010, pp. 37-43.

[6] http://openid.net. 